\title{
Statistical Inferences Related to the Threshold Values of Uniform and Exponential Distributions
}

\author{
Prof. Dr. Ismail ERDEM \\ Baskent University Faculty of Economics and Administrative Sciences \\ Department of Economics \\ Baglica, Ankara, Turkey 06530 \\ iserdem@baskent.edu.tr
}

\begin{abstract}
The estimation of threshold values by various statistical approaches has been intensively studied since 2002. However, confidence intervals and hypothesis testing procedures for the threshold parameters of uniform and exponential distributions have been neglected in threshold value-related inferential studies. The thresholds of random variables with assumed distributions are not always known, and must sometimes be inferred. In this work, the unknown threshold values of uniform and exponential distributions are inferred from unbiased maximum likelihood estimators and their probability density functions. Procedures for constructing confidence intervals and tests of hypotheses related to the unknown threshold values are established. Simulated data are generated from a uniform and an exponential distribution, both with unknown threshold parameters, using the statistical software MINITAB. Based on the simulated data, confidence intervals are constructed and hypotheses are tested on the unknown threshold values. The simulation results almost perfectly agree with the theoretical analysis. Using the proposed procedures, researchers could estimate the threshold parameters of other distributions in future inference-making studies.
\end{abstract}

Keywords: Uniform and Exponential Distributions, the Distributions of First order Statistics, Unbiased Estimators, Confidence Intervals and Tests of Hypotheses for the threshold values.

\section{INTRODUCTION}

Estimating the various parameters of certain distributions is a popular topic in statistics. For example, the two-parameter exponential distribution (in which one parameter is the threshold value) has been applied to the analysis of lifetime data [1], and the inventory management of hazardous items [2]. Erdem [3] studied inferential procedures for the parameters of triangular distributions, and Petropoulos [4] showed that the first-order statistic is sufficient for determining the threshold parameter of the two-parameter exponential distribution. The intervals of this distribution have also been estimated [5]. Bhushal and Sipakar [6] estimated the threshold point of growth and inflation in Nepal. Massacci [7] estimated unknown thresholds using large-dimensional threshold factor models. The impact of missing thresholds was investigated by Riley et al. [8] in a meta-analysis context. Morfeld et al. [9] estimated the threshold values in the distributions of respirable quartz dust concentration and silicosis incidence in a cohort of German porcelain workers. Mann [10] stated that "Earth will cross the climate danger threshold by 2036". However, to my knowledge, interval estimation and hypotheses testing of the threshold parameters of uniform and exponential distributions has not been reported.

\subsection{Uniform and Exponential Distributions with Unknown Threshold Values}

Exponential and uniform distributions are commonly encountered in physical situations. For example, the amount of gasoline sold daily at a service station may be uniformly distributed between a minimum and maximum of 2,000 and 5,000 gallons, respectively. Similarly, the waiting time of a passenger at a subway station may uniformly vary between 1 and 15 minutes.

Examples of random variables following exponential distributions are the length of time between telephone calls (or between arrivals at a service station), and the lifetimes of electronic components (i.e., time to internal failure).

In this work, the unknown threshold parameters of uniform and exponential distributions are estimated from unbiased maximum likelihood estimators (MLEs) and their probability density 
functions. Procedures for constructing the confidence intervals and tests of hypotheses related to these unknown threshold parameters are also established. To date, such procedures have not been reported for the threshold parameters of these distributions.

The threshold parameter of a random variable is not always known. However, as the threshold parameter $\theta$ provides an estimate of the earliest time to failure (or success), it is an important parameter of random variables, and may need to be inferred.

To estimate the unknown parameter of interest, a random sample of size $n$ is taken from the distribution (population). In general, the MLE provides a plausible estimator. The MLEs of the threshold parameters of uniform and exponential distributions constitute the first-order statistics in the present study.

This work presents unbiased estimators for the threshold parameters of the uniform and exponential distributions, and establishes procedures for constructing confidence intervals and testing hypotheses of these parameters. The theoretical findings are validated in simulation studies of data generated by the statistical software MINITAB. The simulation results are summarized in Section 6, and presented in full (along with the computations) in the Appendices.

\subsection{Probability Density Function (Pdf) and Cumulative Distribution Function (Cdf) of the First-Order Statistic}

Let $X_{1}, X_{2}, \ldots, X_{n}$ be a random sample of size $n$ from a continuous distribution over the interval $(c, d)$, and let $Y_{i}$ be the $i$ th-order statistic $(i=1,2, \ldots n)$.

The formula for computing the pdf of the ith-order statistic is found in almost all mathematical statistics textbooks, including Roussas [11]:

$$
f_{Y_{i}}(y)=\frac{n !}{(i-1) !(n-i) !}[F(y)]^{(i-1)}[1-F(y)]^{(n-i)} f_{Y}(y) \quad, c<y<d,
$$

where $n$ is the size of the random sample, $F(y)$ is the cumulative distribution function (cdf), and $f_{Y_{i}}(y)$ is the pdf of the $i$ th-order statistic $Y_{i}$.

When $i=1$, the pdf reduces to:

$$
f_{Y_{1}}(y)=n[1-F(y)]^{(n-1)} f_{Y}(y), c<y<d
$$

The cumulative distribution function (cdf) of the first-order statistic is given by

$$
F(y)=P\left(Y_{1} \leq y\right)=\int_{c}^{y} n[1-F(y)]^{(n-1)} f_{Y}(y) d y=[1-F(c)]^{(n)}-[1-F(y)]^{(n)}
$$

\subsection{Properties of the First-Order Statistic From $X \sim \operatorname{Uniform}(\theta, b)$}

Assuming real values in the interval $(\theta, b)$, a uniformly-distributed continuous random variable has the pdf

$$
f(x)=\frac{1}{b-\theta}, \theta<x<b,
$$

Where $b$ is the known upper bound value, and $\theta$ is the unknown threshold parameter of the uniformly distributed random variable $X$.

The MLE of the threshold parameter $\theta$ is the first-order statistic of a random sample of size $n$, namely, $Y_{1}=\operatorname{Min}\left\{X_{1}, X_{2}, \ldots, X_{n}\right\}$.

The pdf given by (4) has the following cdf:

$$
F(y)=\int_{\theta}^{y} \frac{1}{b-\theta} d x=\frac{y-\theta}{b-\theta}, \quad \theta<y<b .
$$


Equations (2) give the following pdf for $Y_{1}$ :

$$
f_{Y_{1}}(y)=n\left[1-\frac{y-\theta}{b-\theta}\right]^{(n-1)}\left(\frac{1}{b-\theta}\right)=\frac{n}{(b-\theta)^{n}}(b-y)^{(n-1)}, \theta<y<b .
$$

The expected value and variance of $Y_{1}$ is then computed as follows:

$$
E\left(Y_{1}^{k}\right)=\frac{n}{(b-\theta)^{n}} \int_{\theta}^{b} y^{k}(b-y)^{(n-1)} d y
$$

where $\mathrm{k}$ is the order of the moment of $Y_{1}(k=1,2)$.

$$
E\left(Y_{1}\right)=\frac{n \theta+b}{(n+1)}, E\left(Y_{1}^{2}\right)=\frac{n(b-\theta)^{2}}{(n+2)}, \operatorname{Var}\left(Y_{1}\right)=\frac{n(b-\theta)^{2}}{(n+1)^{2}(n+2)}
$$

From (7) the unbiased estimator of $\theta$ is given as

$$
\begin{aligned}
& T_{1}=\frac{(n+1) Y_{1}-b}{n} \rightarrow E\left(T_{1}\right)=\theta \\
& \operatorname{Var}\left(T_{1}\right)=\left(\frac{n+1}{n}\right)^{2} \operatorname{Var}\left(Y_{1}\right)=\frac{(b-\theta)^{2}}{n(n+2)} .
\end{aligned}
$$

2. Confidence Interval for the Threshold Parameter $\theta$ OF The Distribution $X \sim \operatorname{Uniform}(\theta, b)$

From the following probability statements, we construct a $100(1-\alpha) \%$ confidence interval for $\theta$ as (where $\alpha$ denotes the level of significance)

$$
P\left(y_{1 L}<Y_{1}<y_{1 U}\right)=(1-\alpha) \text {. }
$$

Equivalently we can write,

$$
F\left(y_{1 L}\right)=P\left(Y_{1}<y_{1 L}\right)=\frac{\alpha}{2}, \text { and } F\left(y_{1 U}\right)=P\left(Y_{1}<y_{1 U}\right)=1-\frac{\alpha}{2} \text {. }
$$

From Eq. (5), we obtain

$1-F(y)=1-\frac{y-\theta}{b-\theta}=\frac{b-y}{b-\theta}, \quad \theta<y<b$.

Substituting this expression into (3), we have

$$
F\left(y_{1 L}\right)=P\left(Y_{1}<y_{1 L}\right)=\left[\frac{b-\theta}{b-\theta}\right]^{n}-\left[\frac{b-y_{1 L}}{b-\theta}\right]^{n}=1-\left[\frac{b-y_{1 L}}{b-\theta}\right]^{n}=\frac{\alpha}{2},
$$

from which $y_{1 L}$ is obtained as

$$
y_{1 L}=b-(b-\theta)\left(1-\frac{\alpha}{2}\right)^{1 / n}
$$

Similarly,

$$
\begin{aligned}
& F\left(y_{1 U}\right)=P\left(Y_{1}<y_{1 U}\right)=\left[\frac{b-\theta}{b-\theta}\right]^{n}-\left[\frac{b-y_{1 U}}{b-\theta}\right]^{n}=1-\left[\frac{b-y_{1 U}}{b-\theta}\right]^{n}=1-\frac{\alpha}{2} \\
& y_{1 U}=b-(b-\theta)\left(\frac{\alpha}{2}\right)^{1 / n} .
\end{aligned}
$$


Substituting the results of (11) and (12) into (10), we obtain

$$
P\left(b-(b-\theta)\left(1-\frac{\alpha}{2}\right)^{1 / n}<Y_{1}<b-(b-\theta)\left(\frac{\alpha}{2}\right)^{1 / n}\right)=(1-\alpha) \text {. }
$$

Solving the above inequalities for $\theta$, the $(1-\alpha) 100 \%$ confidence interval (in terms of the first order statistic $Y_{1}$ ) of the threshold parameter $\theta$ is obtained as

$$
\left(b-\frac{b-Y_{1}}{\left(\frac{\alpha}{2}\right)^{1 / n}}, b-\frac{b-Y_{1}}{\left(1-\frac{\alpha}{2}\right)^{1 / n}}\right)
$$

This confidence interval can also be expressed in terms of the unbiased estimate $T_{1}$ of the threshold parameter $\theta$ :

$$
\left(b-\frac{n\left(b-T_{1}\right)}{(n+1)\left(\frac{\alpha}{2}\right)^{1 / n}}, \quad b-\frac{n\left(b-T_{1}\right)}{(n+1)\left(1-\frac{\alpha}{2}\right)^{1 / n}}\right) .
$$

\section{Tests of Hypotheses Related to the Threshold Value $\theta$ of the Random VARIABLE $X \sim$ Uniform $(\theta, b)$}

When testing $H_{0}: \theta=\theta_{0}, H_{0}: \theta \leq \theta_{0}$ or $H_{0}: \theta \geq \theta_{0}$ against the proper alternative hypothesis, a plausible test statistic is $Y_{1}=X_{\text {Min }}$, for which the unbiased estimator of $\theta$ (given by Eq. (1.8)) is a linear function of $Y_{1}$.

For the chosen significance level $\alpha$, the following decision rules apply.

Table 1. Tests of Hypotheses Related to the Threshold value $\theta$ for the Distribution $X \sim$ Uniform $(\theta, b)$

\begin{tabular}{|l|l|l|}
\hline $\begin{array}{l}H_{0}: \theta=\theta_{0} \\
H_{1}: \theta \neq \theta_{0}\end{array}$ & $\begin{array}{l}H_{0}: \theta \leq \theta_{0} \\
H_{1}: \theta>\theta_{0}\end{array}$ & $\begin{array}{l}H_{0}: \theta \geq \theta_{0} \\
H_{1}: \theta<\theta_{0}\end{array}$ \\
\hline $\begin{array}{l}\text { If } Y_{1} \geq y_{1 U} \text { or } Y_{1} \leq y_{1 L} \\
H_{0} \text { is rejected }\end{array}$ & If $Y_{1} \geq y_{1 U} H_{0}$ is rejected & If $Y_{1} \leq y_{1 L} H_{0}$ is rejected \\
\hline Don't reject $\mathrm{H}_{0}$ otherwise & Don't reject $\mathrm{H}_{0}$ otherwise & Don't reject $\mathrm{H}_{0}$ otherwise \\
\hline Where, $y_{1 L}=b-\left(b-\theta_{0}\right)\left(1-\frac{\alpha}{2}\right)^{1 / n}$ and & $\begin{array}{l}\text { Where, } \\
y_{1 U}=b-\left(b-\theta_{0}\right)(\alpha)^{1 / n}\end{array}$ & $\begin{array}{l}\text { Where } \\
y_{1 L}=b-\left(b-\theta_{0}\right)(1-\alpha)^{1 / n}\end{array}$ \\
$y_{1 U}=b-\left(b-\theta_{0}\right)\left(\frac{\alpha}{2}\right)^{1 / n}$ & & \\
\hline
\end{tabular}

Note that in the procedures for constructing confidence intervals and testing hypotheses, we include the observed value of the first order statistic $Y_{1}$ and the chosen significance level $\alpha$.

\section{Confidence Interval For the Threshold Parameter $\beta$ OF THE EXPonential DISTRIBUTION}

Let $Y$ be an exponentially distributed continuous random variable with an unknown threshold parameter $\beta$ and a known parameter $\lambda$. The pdf of $Y$ is given by 


$$
\begin{aligned}
& f_{Y}(y ; \beta, \lambda)=e^{\beta / \lambda} \frac{1}{\lambda} e^{-y / \lambda} ; y>\beta>0, \lambda>0 \\
& F(y)=P(Y \leq y)=e^{\beta / \lambda} \int_{\beta}^{y} \frac{1}{\lambda} e^{-y / \lambda} d t=1-e^{\beta / \lambda} e^{-y / \lambda}, y>\beta>0 \rightarrow \\
& 1-F(y)=1-P(Y \leq y)=e^{\beta / \lambda} e^{-y / \lambda}, y>\beta>0
\end{aligned}
$$

The MLE of $\beta$ is the first-order statistic of a random sample of size $n$.

Let $W_{1}$ be the first-order statistic of a random sample of size $n$ from this distribution. The pdf of $W_{1}$ is given by

$$
\begin{aligned}
& f_{W_{1}}(w, \beta, \lambda)=e^{n \beta / \lambda} \frac{n}{\lambda} e^{-n w / \lambda}, w>\beta>0, \\
& E\left(W_{1}\right)=\int_{\beta}^{\infty} w e^{n \beta / \lambda} \frac{n}{\lambda} e^{-n w / \lambda} d w=\beta+\frac{\lambda}{n}, \\
& E\left(W_{1}^{2}\right)=\int_{\beta}^{\infty} w^{2} e^{n \beta / \lambda} \frac{n}{\lambda} e^{-n w / \lambda} d w=\left(\beta+\frac{\lambda}{n}\right)^{2}+\frac{\lambda^{2}}{n^{2}} \\
& \operatorname{Var}\left(W_{1}\right)=\frac{\lambda^{2}}{n^{2}}
\end{aligned}
$$

From (17) we can derive an unbiased estimator for the unknown threshold parameter $\beta$.

$$
T_{2}=W_{1}-\frac{\lambda}{n}, \quad \operatorname{Var}\left(T_{2}\right)=\operatorname{Var}\left(W_{1}-\frac{\lambda}{n}\right)=\operatorname{Var}\left(W_{1}\right)=\frac{\lambda^{2}}{n^{2}}
$$

From the probability expression $P\left(w_{L}<W_{1}<w_{U}\right)=1-\alpha$, we can determine a $100(1-\alpha) \%$ confidence interval for $\beta$. The above probability expression may also be expressed as

$$
P\left(W_{1}<w_{L}\right)=\frac{\alpha}{2} \text { and } P\left(W_{1}>w_{U}\right)=\frac{\alpha}{2} .
$$

Using Eqs. (3) and (16), we obtain

$$
P\left(W_{1}<w_{L}\right)=-\left.\left[e^{\beta / \lambda} e^{-y / \lambda}\right]_{\beta}^{w_{L}}\right|_{\beta}=1-\left[e^{\beta / \lambda} e^{-w_{L} / \lambda}\right]^{n}=\frac{\alpha}{2},
$$

from which $w_{L}$ is obtained as

$$
w_{L}=\beta-\frac{\lambda}{n} \ln \left(1-\frac{\alpha}{2}\right) \text {. }
$$

Similarly,

$$
\left.P\left(W_{1}<w_{U}\right)=-\left[e^{\beta / \lambda} e^{-y / \lambda}\right]\right]\left._{\beta}^{n}\right|_{\beta} ^{w_{U}}=1-\left[e^{\beta / \lambda} e^{-w_{U} / \lambda}\right]^{n}=1-\frac{\alpha}{2} .
$$

From (22), we obtain

$$
w_{U}=\beta-\frac{\lambda}{n} \ln \left(\frac{\alpha}{2}\right)
$$

Inserting (21) and (23) in to $P\left(w_{L}<W_{1}<w_{U}\right)=1-\alpha$, we obtain the intended confidence interval for the threshold parameter $\beta$; namely, 


$$
P\left[w_{1}+\frac{\lambda}{n} \ln (\alpha / 2)<\beta<w_{1}+\frac{\lambda}{n} \ln (1-\alpha / 2)\right]=(1-\alpha)
$$

This confidence interval may also be expressed in terms of the unbiased estimate $T_{2}$ of the threshold $\beta$

$$
\begin{aligned}
& T_{2}=\left(w_{1}-\frac{\lambda}{n}\right) \rightarrow w_{1}=\left(T_{2}+\frac{\lambda}{n}\right) ; \text { that is, } \\
& P\left(T_{2}+\frac{\lambda}{n}(1+\ln (\alpha / 2))<\beta<T_{2}+\frac{\lambda}{n}(1+\ln (1-\alpha / 2))\right)=(1-\alpha) .
\end{aligned}
$$

\section{Hypotheses Tests Related to the Threshold Parameter $\beta$ OF the EXPONENTIAL DISTRIBUTION}

When testing $H_{0}: \beta=\beta_{0}, H_{0}: \beta \leq \beta_{0}$ or $H_{0}: \beta \geq \beta_{0}$ against to the proper alternative hypothesis, a plausible test statistic is $W_{1}=X_{\text {Min }}$.

For the chosen significance level $\alpha$, the decision rules listed in Table 2 are applicable.

Table 2. Tests of Hypotheses Related to the Threshold Value $\beta$ of the Distribution $W \sim \operatorname{Exponential}(\lambda ; \beta)$

\begin{tabular}{|l|l|l|}
\hline$H_{0}: \beta=\beta_{0}$ & $H_{0}: \beta \leq \beta_{0}$ & $H_{0}: \beta \geq \beta_{0}$ \\
$H_{1}: \beta \neq \beta_{0}$ & $H_{1}: \beta>\beta_{0}$ & $H_{1}: \beta<\beta_{0}$ \\
\hline $\begin{array}{l}\text { If } W_{1} \geq w_{U} \text { or } W_{1} \leq w_{L}, \\
\text { reject } H_{0}\end{array}$ & If $W_{1} \geq w_{U}$, reject $H_{0}$ & If $W_{1} \leq w_{L}$, reject $H_{0}$ \\
\hline Otherwise, do not reject $\mathrm{H}_{0}$ & Otherwise, do not reject $\mathrm{H}_{0}$ & Otherwise, do not reject $\mathrm{H}_{0}$ \\
\hline where & where & where \\
$w_{L}=\beta_{0}-\frac{\lambda}{n} \ln \left(1-\frac{\alpha}{2}\right)$ and & $w_{U}=\beta_{0}-\frac{\lambda}{n} \ln (\alpha)$ & $w_{L}=\beta_{0}-\frac{\lambda}{n} \ln (1-\alpha)$ \\
$w_{U}=\beta_{0}-\frac{\lambda}{n} \ln \left(\frac{\alpha}{2}\right)$ & & \\
\hline
\end{tabular}

\section{Simulation STUdieS}

To validate the theoretical findings of the studied distributions, I simulated a uniform distribution with threshold parameter $\theta$ over the interval $(\theta=5, b=10)$, and on an exponential distribution with threshold parameter $\beta=4$ and $\lambda=0.2$.

It is a known fact that, for any distribution, the cumulative distribution function $F(y)$ is a random variable with a uniform distribution over the interval $(0,1)$.

Using MINITAB [12] software, 100 independent random samples of sizes 100 were drawn from a uniform distribution over the interval $(0,1)$. These observations were considered as the sampled $F(y)$ values.

To obtain corresponding random observations from a uniform distribution over the interval $(\theta, b)$, I applied an inverse transformation to the cdf. The $[F(y)]$ of this distribution becomes

$$
F(y)=\frac{y-\theta}{b-\theta} \rightarrow y=\theta+(b-\theta) F(y) .
$$

The sampled $F(y)$ values were used to simulate the uniform distribution over the interval $(\theta=5, b=10)$. Equation (26) yields $y=5+(10-5) F(y)=5+5 F(y)$ values. From each random sample of size 100 , the first-order statistic $y_{1}$ was determined, and unbiased estimator values were 
computed. The simulation results are summarized in Table 3. As indicated in the table, $E\left(T_{1}\right) \cong \theta=5$ with a very small coefficient of variation.

[Coefficient of Variation for $T_{1}=0.00289 / 5.00116 \approx 5.78 \times 10^{-4}$ ].

Table 3. Simulation Summaries for Uniform Distribution

\begin{tabular}{|l|l|l|}
\hline & Mean & Variance \\
\hline$Y_{1}$ & $\mathbf{5 . 0 5 0 6 5}$ & $\mathbf{0 . 0 1 8 0 6}$ \\
\hline $\mathrm{T}_{1}$ & $\mathbf{5 . 0 0 1 1 6}$ & $\mathbf{0 . 0 0 2 8 9}$ \\
\hline Lower Confidence Limit (LCL) & $\mathbf{4 . 6 9 9 6 8}$ & \\
\hline Upper Confidence Limit (UCL) & $\mathbf{5 . 0 4 9 4}$ & \\
\hline
\end{tabular}

From each random sample of size 100, the $95 \%$ confidence intervals of $\theta$ were computed by the rule derived from (14). Ninety-seven of these 100 confidence intervals contained the true value $(=5)$ of the threshold parameter $\theta$.

Table 4. Simulation summaries for the hypotheses tests Related to the threshold parameter of the random variable $X \sim$ Uniform $(\theta=5, b=10)$

$$
\begin{array}{l|l}
H_{0}: \theta=5 & y_{1 L}=10-(10-5)(0.975)^{1 / 100} \cong 5.001266 \\
H_{1} \theta \neq 5 & y_{1 U}=10-(10-5)(0.025)^{1 / 100} \cong 5.18108 \\
\text { If } Y_{1} \geq y_{1 U} \text { or } Y_{1} \leq y_{1 L} H_{0} \text { is rejected } &
\end{array}
$$

In 6 out of 100 tests, $H_{0}: \theta=5$ was rejected at the $\alpha=0.05$ significance level.

For ease of visualization, the proportions of accepted confidence intervals and hypotheses after the simulations are graphically presented in Fig. 1. The charts summarize the results of the uniform distribution.

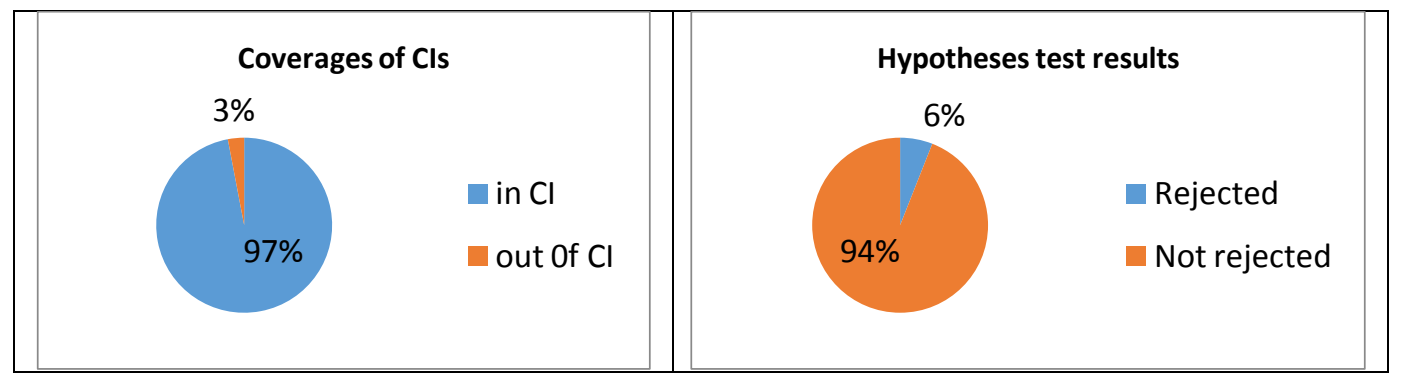

Fig. 1. Pie charts showing the proportions of accepted and rejected confidence intervals (CI) and hypotheses in the simulated uniform distribution.

The hypotheses in Table 4 were tested on the same simulated raw data described at the beginning of this section. The decisions were classified into rejecting or not rejecting the null hypotheses. At the chosen level of significance $(\alpha=0.05)$, the true null hypothesis was rejected in only 6 out of 100 tests.

Similar procedures were followed for the threshold parameter $\beta$ of the exponential distribution. One hundred samples of size 100 were generated from the uniform distribution over the interval $(0,1)$. These were converted into 100 samples from an exponential distribution with the probability density function $f_{Y}(y ; \beta, \lambda)=e^{\beta / \lambda} \frac{1}{\lambda} e^{-y / \lambda}, y>\beta>0, \lambda>0$ by an inverse transformation applied on the following cumulative probability distribution function $F(y)=1-e^{\beta / \lambda} e^{-y / \lambda}, y>\beta>0$. The transformation procedure is described below.

Let $F\left(y_{i}\right)=u_{i}$ be a randomly generated observation from a uniform distribution over the interval

$(0,1)$. To convert this to a random observation from the exponential distribution, we set $u_{i}=1-e^{\beta / \lambda} e^{-y_{i} / \lambda}$, yielding

$y_{i}=\beta-\lambda \ln \left(1-u_{i}\right)$. 
Inserting $\lambda=0.2$ and $\beta=4$ into (27), the required observations are generated through $y_{i}=4-0.2 \ln \left(1-u_{i}\right)$. From each sample of size 100 , we determine the smallest observed (simulated) values (the $w_{i}$ values).

The simulation results are summarized in Table 5. According to this table, $E\left(T_{2}\right) \cong \beta=4$ with a very small coefficient of variation.

[Coefficient of Variation for $T_{2}=0.00000304 / 3.999968 \approx 7.60 \times 10^{-7}$ ].

The $95 \%$ confidence intervals of $\beta$ were computed by (4.10). Ninety-seven out of 100 confidence intervals contained the chosen threshold $(\beta=4)$.

Table 5. Simulation summaries for exponential distribution

\begin{tabular}{|l|l|l|}
\hline & Mean & Variance \\
\hline $\mathrm{W}_{1}$ & $\mathbf{4 . 0 0 1 9 0 1}$ & $\mathbf{0 . 0 0 0 0 0 3 0 4}$ \\
\hline $\mathrm{T}_{2}$ & $\mathbf{3 . 9 9 9 9 6 8}$ & $\mathbf{0 . 0 0 0 0 0 3 0 4}$ \\
\hline Lower Confidence Limit (LCL) & $\mathbf{3 . 9 9 4 5 9 1}$ & \\
\hline Upper Confidence Limit (UCL) & $\mathbf{4 . 0 0 1 9 1 8}$ & \\
\hline
\end{tabular}

Table 6. Simulation summaries for the hypotheses tests related to the threshold value of the random variable $W \sim \operatorname{Exponential}(\lambda=0,2 ; \beta=4)$

\begin{tabular}{|l|l|}
\hline$H_{0}: \beta=4$ & where $w_{L}=4-\frac{0.2}{100} \ln (0.975) \equiv 4.000050636$ \\
$H_{1}: \beta \neq 4$ & $w_{U}=4-\frac{0.2}{100} \ln (0.025) \cong 4.007377759$ \\
If $W_{1} \geq w_{U}$ or $W_{1} \leq w_{L}$, & \\
reject $H_{0}$ & \\
\hline$H_{0}: \beta=4$ was rejected in 3 out of 100 tests \\
\hline
\end{tabular}

The confidence intervals and hypothesis test results after the simulations are graphically presented in Fig. 2.

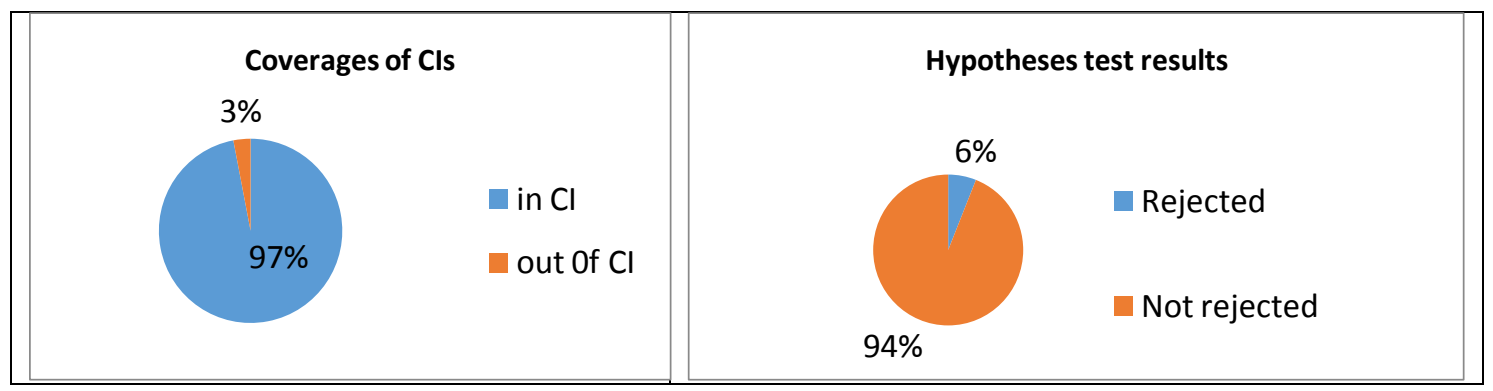

Fig. 2. Pie charts showing the proportions of accepted and rejected confidence intervals (CI) and hypotheses in the simulated exponential distribution.

The hypotheses in Table 6 were tested on the same simulated raw data described at the beginning of this section. Again, the decisions are classified into rejecting or not rejecting the null hypotheses. At the chosen significance level $(\alpha=0.05)$, the true null hypothesis was rejected in only 3 out of 100 tests.

The observed values from the uniform distributions were simulated in the MINITAB [12] package. The detailed computations are tabulated in Appendices I and II.

\section{CONClusion}

This study obtained the unbiased MLEs in uniform and exponential distributions. Procedures for determining the confidence intervals and hypotheses tests were established from the pdfs and cdfs of these estimators. The developed procedures might also infer the threshold parameters of other distributions. Although this study investigated the unknown lower bound threshold of the random variable, the method is equally applicable to the upper bound of the random variable of interest. 
Inferencing of threshold parameters will significantly contribute to decision making problems in real life situations. For instance, when issuing a warranty statement on a machine component, one needs to know the shortest lifetime of the component. If this is unknown but the component lifetime follows a certain distribution (such as uniform or exponential), the above procedures may assist the decision maker in estimating the threshold value, and determining the pdf and cdf of the random variable of interest. Thus, the developed procedures provide robust and reliable information for composing the warranty statement.

As another example, when deciding whether an athlete qualifies for an Olympic 100-m dash race, the committee needs to determine the upper bound of the qualifying completion time. For this purpose, they may estimate the upper confidence limit of the threshold parameter from the 100-m dash completion times of the world's top competitors in recent past events (such as the last 5 Olympics).

\section{ACKNOWLEDGEMENTS}

I owe many thanks to Baskent University administration for supporting this study.

\section{REFERENCES}

[1] Lawless, J.F., Prediction intervals for the two-parameter exponential distribution, Technimetrics, vol. 19, no.4, pp.469-472, (1977).

[2] Baten, A. and Kamil, A., Inventory Management systems with hazardous items of two-parameter exponential distribution, Journal of Social Sciences, vol. 5, pp. 183-187, (2009).

[3] Erdem, I., Statistical Inferences on Type I and Type II Triangular Distributions, International Journal of Applied Science and Technology, Vol. 2, No 1; January (2012).

[4] Petropoulos, C., New Classes of Improved Confidence intervals for the Scale Parameter of a two-parameter exponential distribution, Statistical Methodology, vol. 8, no. 4, pp. 401-410, (2011).

[5] Jiang L. and Wong, A.C.M., An Interval Estimation of the Two-Parameter Exponential Distribution, Journal Probability and Statistics, Volume 2012(2012), http://dx.doi.org/10.155/2012/734575.

[6] Bhusal, T.P. and Silpakar, S., Growth and Inflation: Estimation of the Threshold Point for Nepal, Economic Journal of Development Issues, vol. 13\&14No. 1-2 (2011) Combined issue.

[7] Massacci, D., Least Square Estimation of Large Dimensional Threshold Factor Models, Social Science Research Network, September 8, (2015).

[8] Riley, R.D., Ahmad, I., Ensor, J., Takwoingi, Y., Kirkham, A., Morris, R.K., Noordzij, J. P. and Deks, J., Meta-Analysis of test accuracy studies: an exploratory method for investigating the impact of missing threshold, Systematic Reviews, (2015), 4:12. Doi: 10.1186/2046-4053-4-12.

[9] Morfeld, P., Mundt, K.A., Taeger, D., Gulner, K., Steinig,O. and Miller, G.B., Threshold value estimation for respirable quartz dust exposure and silicosis incidence among workers in the German porcelain industry, Journal of Occupational Environment Medicine, (2013). Sep.: 55(9):1027-34 doi:10.1097/JOM.0b013e318298327a.

[10] Mann, E., Earth Will Cross the Climate Danger Threshold by 2036. Scientific American, March 18, (2014), Volume 310, Issue 4,

[11] Roussas, G.G., A First Course in Mathematical Statistics, Addison Wesley Publishing Company, 1973, pp: 194-195

[12] MINITAB 17, Minitab Statistical Software. (www.minitab.com)

\section{AUTHOR's BIOGRAPHY}

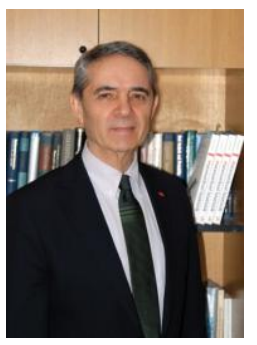

Ismail Erdem, Ph.D. from North Carolina State University (USA). Has quite lengthy teaching/administrative experience. Worked for North Carolina State and North Carolina Central Universities for 5 years. Later, worked for Middle East Technical University (METU) Ankara/Turkey for 16 years. After METU, joined Baskent University Ankara-Turkey, has been teaching statistics and quantitative methods for 39 years. Main interest areas are: Mathematical and Applied Statistics, Time Series Analysis, and Forecasting. He has more than 40 National/International publications in science journals and conference materials. 


\section{APPENDIX I}

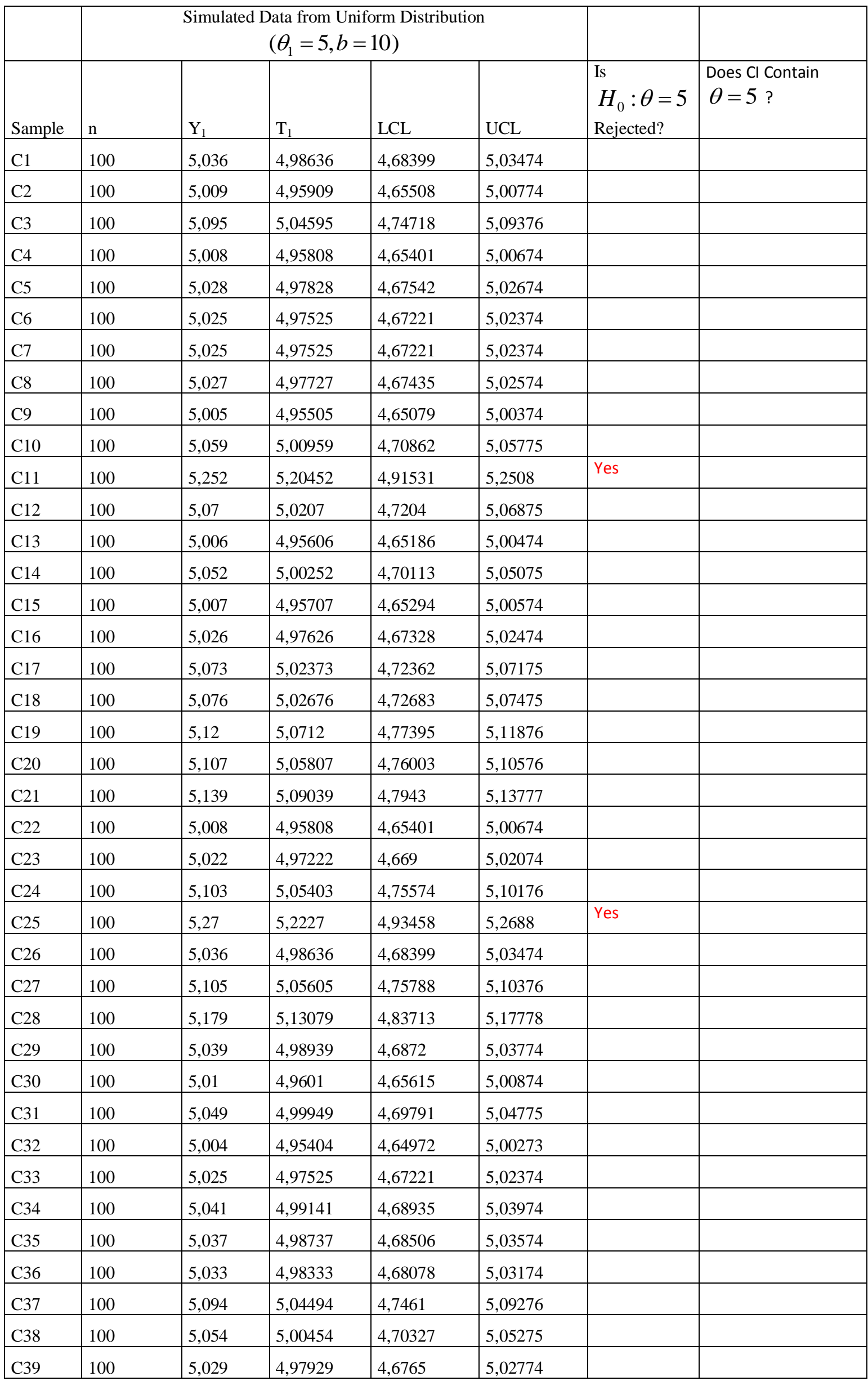


Statistical Inferences Related to the Threshold Values of Uniform and Exponential Distributions

\begin{tabular}{|c|c|c|c|c|c|c|c|}
\hline $\mathrm{C} 40$ & 100 & 5 & 4,95 & 4,64544 & 4,99873 & Yes & No \\
\hline C41 & 100 & 5,019 & 4,96919 & 4,66579 & 5,01774 & & \\
\hline $\mathrm{C} 42$ & 100 & 5,003 & 4,95303 & 4,64865 & 5,00173 & & \\
\hline $\mathrm{C} 43$ & 100 & 5,033 & 4,98333 & 4,68078 & 5,03174 & & \\
\hline C44 & 100 & 5,1 & 5,051 & 4,75253 & 5,09876 & & \\
\hline $\mathrm{C} 45$ & 100 & 5,062 & 5,01262 & 4,71184 & 5,06075 & & \\
\hline $\mathrm{C} 46$ & 100 & 5,024 & 4,97424 & 4,67114 & 5,02274 & & \\
\hline $\mathrm{C} 47$ & 100 & 5,058 & 5,00858 & 4,70755 & 5,05675 & & \\
\hline $\mathrm{C} 48$ & 100 & 5,06 & 5,0106 & 4,70969 & 5,05875 & & \\
\hline C49 & 100 & 5,062 & 5,01262 & 4,71184 & 5,06075 & & \\
\hline C50 & 100 & 5,043 & 4,99343 & 4,69149 & 5,04174 & & \\
\hline C51 & 100 & 5,036 & 4,98636 & 4,68399 & 5,03474 & & \\
\hline $\mathrm{C} 52$ & 100 & 5,038 & 4,98838 & 4,68613 & 5,03674 & & \\
\hline C53 & 100 & 5,011 & 4,96111 & 4,65722 & 5,00974 & & \\
\hline C54 & 100 & 5,014 & 4,96414 & 4,66043 & 5,01274 & & \\
\hline C55 & 100 & 5,016 & 4,96616 & 4,66257 & 5,01474 & & \\
\hline C56 & 100 & 5,016 & 4,96616 & 4,66257 & 5,01474 & & \\
\hline C57 & 100 & 5,056 & 5,00656 & 4,70541 & 5,05475 & & \\
\hline C58 & 100 & 5,005 & 4,95505 & 4,65079 & 5,00374 & & \\
\hline C59 & 100 & 5,065 & 5,01565 & 4,71505 & 5,06375 & & \\
\hline C60 & 100 & 5,069 & 5,01969 & 4,71933 & 5,06775 & & \\
\hline C61 & 100 & 5,01 & 4,9601 & 4,65615 & 5,00874 & & \\
\hline C62 & 100 & 5,001 & 4,95101 & 4,64651 & 4,99973 & Yes & No \\
\hline C63 & 100 & 5,007 & 4,95707 & 4,65294 & 5,00574 & & \\
\hline C64 & 100 & 5,066 & 5,01666 & 4,71612 & 5,06475 & & \\
\hline C65 & 100 & 5,009 & 4,95909 & 4,65508 & 5,00774 & & \\
\hline C66 & 100 & 5,022 & 4,97222 & 4,669 & 5,02074 & & \\
\hline C67 & 100 & 5,131 & 5,08231 & 4,78573 & 5,12977 & & \\
\hline C68 & 100 & 5,024 & 4,97424 & 4,67114 & 5,02274 & & \\
\hline C69 & 100 & 5,01 & 4,9601 & 4,65615 & 5,00874 & & \\
\hline C70 & 100 & 5,004 & 4,95404 & 4,64972 & 5,00273 & & \\
\hline C71 & 100 & 5,001 & 4,95101 & 4,64651 & 4,99973 & Yes & No \\
\hline C72 & 100 & 5,123 & 5,07423 & 4,77716 & 5,12177 & & \\
\hline C73 & 100 & 5,019 & 4,96919 & 4,66579 & 5,01774 & & \\
\hline C74 & 100 & 5,128 & 5,07928 & 4,78252 & 5,12677 & & \\
\hline C75 & 100 & 5,044 & 4,99444 & 4,69256 & 5,04275 & & \\
\hline C76 & 100 & 5,014 & 4,96414 & 4,66043 & 5,01274 & & \\
\hline C77 & 100 & 5,17 & 5,1217 & 4,82749 & 5,16878 & & \\
\hline C78 & 100 & 5,031 & 4,98131 & 4,67864 & 5,02974 & & \\
\hline C79 & 100 & 5,012 & 4,96212 & 4,65829 & 5,01074 & & \\
\hline $\mathrm{C} 80$ & 100 & 5,009 & 4,95909 & 4,65508 & 5,00774 & & \\
\hline C81 & 100 & 5,036 & 4,98636 & 4,68399 & 5,03474 & & \\
\hline C82 & 100 & 5,025 & 4,97525 & 4,67221 & 5,02374 & & \\
\hline $\mathrm{C} 83$ & 100 & 5,109 & 5,06009 & 4,76217 & 5,10776 & & \\
\hline
\end{tabular}


Prof. Dr. Ismail ERDEM

\begin{tabular}{|c|c|c|c|c|c|c|c|}
\hline $\mathrm{C} 84$ & 100 & 5,032 & 4,98232 & 4,67971 & 5,03074 & & \\
\hline $\mathrm{C} 85$ & 100 & 5,156 & 5,10756 & 4,8125 & 5,15477 & & \\
\hline $\mathrm{C} 86$ & 100 & 5,012 & 4,96212 & 4,65829 & 5,01074 & & \\
\hline $\mathrm{C} 87$ & 100 & 5,062 & 5,01262 & 4,71184 & 5,06075 & & \\
\hline $\mathrm{C} 88$ & 100 & 5,008 & 4,95808 & 4,65401 & 5,00674 & & \\
\hline $\mathrm{C} 89$ & 100 & 5,004 & 4,95404 & 4,64972 & 5,00273 & & \\
\hline C90 & 100 & 5,038 & 4,98838 & 4,68613 & 5,03674 & & \\
\hline C91 & 100 & 5,204 & 5,15604 & 4,8639 & 5,20279 & Yes & \\
\hline C92 & 100 & 5,015 & 4,96515 & 4,6615 & 5,01374 & & \\
\hline C93 & 100 & 5,114 & 5,06514 & 4,76752 & 5,11276 & & \\
\hline C94 & 100 & 5,01 & 4,9601 & 4,65615 & 5,00874 & & \\
\hline C95 & 100 & 5,006 & 4,95606 & 4,65186 & 5,00474 & & \\
\hline C96 & 100 & 5,028 & 4,97828 & 4,67542 & 5,02674 & & \\
\hline C97 & 100 & 5,074 & 5,02474 & 4,72469 & 5,07275 & & \\
\hline C98 & 100 & 5,041 & 4,99141 & 4,68935 & 5,03974 & & \\
\hline C99 & 100 & 5,055 & 5,00555 & 4,70434 & 5,05375 & & \\
\hline C100 & 100 & 5,028 & 4,97828 & 4,67542 & 5,02674 & & \\
\hline & Mean & 5,05065 & 5,00116 & 4,69968 & 5,0494 & & \\
\hline & Variance & 0,00284 & 0,00289 & 0,00325 & 0,00284 & & \\
\hline & Min & 5 & 4,95 & 4,64544 & 4,99873 & & \\
\hline & Max. & 5,27 & 5,2227 & 4,93458 & 5,2688 & & \\
\hline
\end{tabular}

The null hypothesis $H_{0}: \theta=5$ is rejected in 6 out of 100 tests.

Three of the $95 \%$ confidence intervals exclude the threshold $\theta_{1}=5$.

\section{APPENDIX II}

\begin{tabular}{|c|c|c|c|c|c|c|}
\hline \multirow[b]{2}{*}{ Sample No } & \multicolumn{4}{|c|}{ Simulation Data from Exponential Distribution $(\beta=4, \lambda=0,2)$} & \multirow[b]{2}{*}{$\begin{array}{l}\text { Is } \\
H_{0}: \beta=5 \\
\text { Rejected? }\end{array}$} & \multirow[b]{2}{*}{$\begin{array}{l}\text { Does CI } \\
\text { Contain } \\
\beta=5 ?\end{array}$} \\
\hline & $\mathrm{n}$ & $\mathrm{W}_{1}$ & LCL & UCL & & \\
\hline $\mathrm{C} 1$ & 100 & 4,003104 & 3,995726 & 4,003053 & & \\
\hline $\mathrm{C} 2$ & 100 & 4,002658 & 3,99528 & 4,002607 & & \\
\hline $\mathrm{C} 3$ & 100 & 4,00201 & 3,994632 & 4,001959 & & \\
\hline $\mathrm{C} 4$ & 100 & 4,001647 & 3,994269 & 4,001596 & & \\
\hline C5 & 100 & 4,00022 & 3,992842 & 4,000169 & & \\
\hline C6 & 100 & 4,004347 & 3,996969 & 4,004296 & & \\
\hline $\mathrm{C7}$ & 100 & 4,007956 & 4,000578 & 4,007906 & Yes & No \\
\hline $\mathrm{C} 8$ & 100 & 4,001647 & 3,994269 & 4,001596 & & \\
\hline $\mathrm{C} 9$ & 100 & 4,001526 & 3,994148 & 4,001475 & & \\
\hline $\mathrm{C} 10$ & 100 & 4,00002 & 3,992642 & 3,999969 & Yes & No \\
\hline $\mathrm{C} 11$ & 100 & 4,00036 & 3,992983 & 4,00031 & & \\
\hline $\mathrm{C} 12$ & 100 & 4,001083 & 3,993705 & 4,001032 & & \\
\hline $\mathrm{C} 13$ & 100 & 4,001687 & 3,994309 & 4,001636 & & \\
\hline $\mathrm{C} 14$ & 100 & 4,000561 & 3,993183 & 4,00051 & & \\
\hline $\mathrm{C} 15$ & 100 & 4,00024 & 3,992862 & 4,00019 & & \\
\hline $\mathrm{C} 16$ & 100 & 4,001143 & 3,993766 & 4,001093 & & \\
\hline $\mathrm{C} 17$ & 100 & 4,003328 & 3,99595 & 4,003277 & & \\
\hline
\end{tabular}


Statistical Inferences Related to the Threshold Values of Uniform and Exponential Distributions

\begin{tabular}{|c|c|c|c|c|c|c|}
\hline $\mathrm{C} 18$ & 100 & 4,002597 & 3,995219 & 4,002546 & & \\
\hline C19 & 100 & 4,001627 & 3,994249 & 4,001576 & & \\
\hline $\mathrm{C} 20$ & 100 & 4,002273 & 3,994895 & 4,002222 & & \\
\hline $\mathrm{C} 21$ & 100 & 4,001345 & 3,993967 & 4,001294 & & \\
\hline $\mathrm{C} 22$ & 100 & 4,002232 & 3,994855 & 4,002182 & & \\
\hline $\mathrm{C} 23$ & 100 & 4,004245 & 3,996867 & 4,004194 & & \\
\hline $\mathrm{C} 24$ & 100 & 4,0003 & 3,992922 & 4,00025 & & \\
\hline $\mathrm{C} 25$ & 100 & 4,002172 & 3,994794 & 4,002121 & & \\
\hline $\mathrm{C} 26$ & 100 & 4,004204 & 3,996826 & 4,004153 & & \\
\hline $\mathrm{C} 27$ & 100 & 4,001546 & 3,994168 & 4,001495 & & \\
\hline $\mathrm{C} 28$ & 100 & 4,002435 & 3,995057 & 4,002384 & & \\
\hline $\mathrm{C} 29$ & 100 & 4,002698 & 3,99532 & 4,002647 & & \\
\hline C30 & 100 & 4,004061 & 3,996683 & 4,00401 & & \\
\hline C31 & 100 & 4,000882 & 3,993504 & 4,000831 & & \\
\hline C32 & 100 & 4,000601 & 3,993223 & 4,00055 & & \\
\hline C33 & 100 & 4,005577 & 3,998199 & 4,005526 & & \\
\hline C34 & 100 & 4,00016 & 3,992782 & 4,000109 & & \\
\hline C35 & 100 & 4,00014 & 3,992762 & 4,000089 & & \\
\hline C36 & 100 & 4,001043 & 3,993665 & 4,000992 & & \\
\hline C37 & 100 & 4,000621 & 3,993243 & 4,00057 & & \\
\hline C38 & 100 & 4,003145 & 3,995767 & 4,003094 & & \\
\hline C39 & 100 & 4,001043 & 3,993665 & 4,000992 & & \\
\hline $\mathrm{C} 40$ & 100 & 4,002496 & 3,995118 & 4,002445 & & \\
\hline C41 & 100 & 4,00286 & 3,995483 & 4,00281 & & \\
\hline $\mathrm{C} 42$ & 100 & 4,005968 & 3,99859 & 4,005918 & & \\
\hline $\mathrm{C} 43$ & 100 & 4,00004 & 3,992662 & 3,999989 & Yes & No \\
\hline $\mathrm{C} 44$ & 100 & 4,00028 & 3,992902 & 4,00023 & & \\
\hline $\mathrm{C} 45$ & 100 & 4,000942 & 3,993564 & 4,000892 & & \\
\hline $\mathrm{C} 46$ & 100 & 4,004654 & 3,997276 & 4,004603 & & \\
\hline $\mathrm{C} 47$ & 100 & 4,000481 & 3,993103 & 4,00043 & & \\
\hline $\mathrm{C} 48$ & 100 & 4,00018 & 3,992802 & 4,000129 & & \\
\hline C49 & 100 & 4,003633 & 3,996255 & 4,003582 & & \\
\hline $\mathrm{C} 50$ & 100 & 4,001224 & 3,993846 & 4,001173 & & \\
\hline C51 & 100 & 4,000461 & 3,993083 & 4,00041 & & \\
\hline $\mathrm{C} 52$ & 100 & 4,00347 & 3,996092 & 4,003419 & & \\
\hline C53 & 100 & 4,005413 & 3,998035 & 4,005362 & & \\
\hline C54 & 100 & 4,002071 & 3,994693 & 4,00202 & & \\
\hline C55 & 100 & 4,001083 & 3,993705 & 4,001032 & & \\
\hline C56 & 100 & 4,000641 & 3,993263 & 4,00059 & & \\
\hline C57 & 100 & 4,008248 & 4,00087 & 4,008197 & & \\
\hline C58 & 100 & 4,001748 & 3,99437 & 4,001697 & & \\
\hline C59 & 100 & 4,005556 & 3,998179 & 4,005506 & & \\
\hline C60 & 100 & 4,001365 & 3,993987 & 4,001314 & & \\
\hline C61 & 100 & 4,004347 & 3,996969 & 4,004296 & & \\
\hline C62 & 100 & 4,00032 & 3,992942 & 4,00027 & & \\
\hline C63 & 100 & 4,001445 & 3,994067 & 4,001395 & & \\
\hline C64 & 100 & 4,00034 & 3,992963 & 4,00029 & & \\
\hline
\end{tabular}


Prof. Dr. Ismail ERDEM

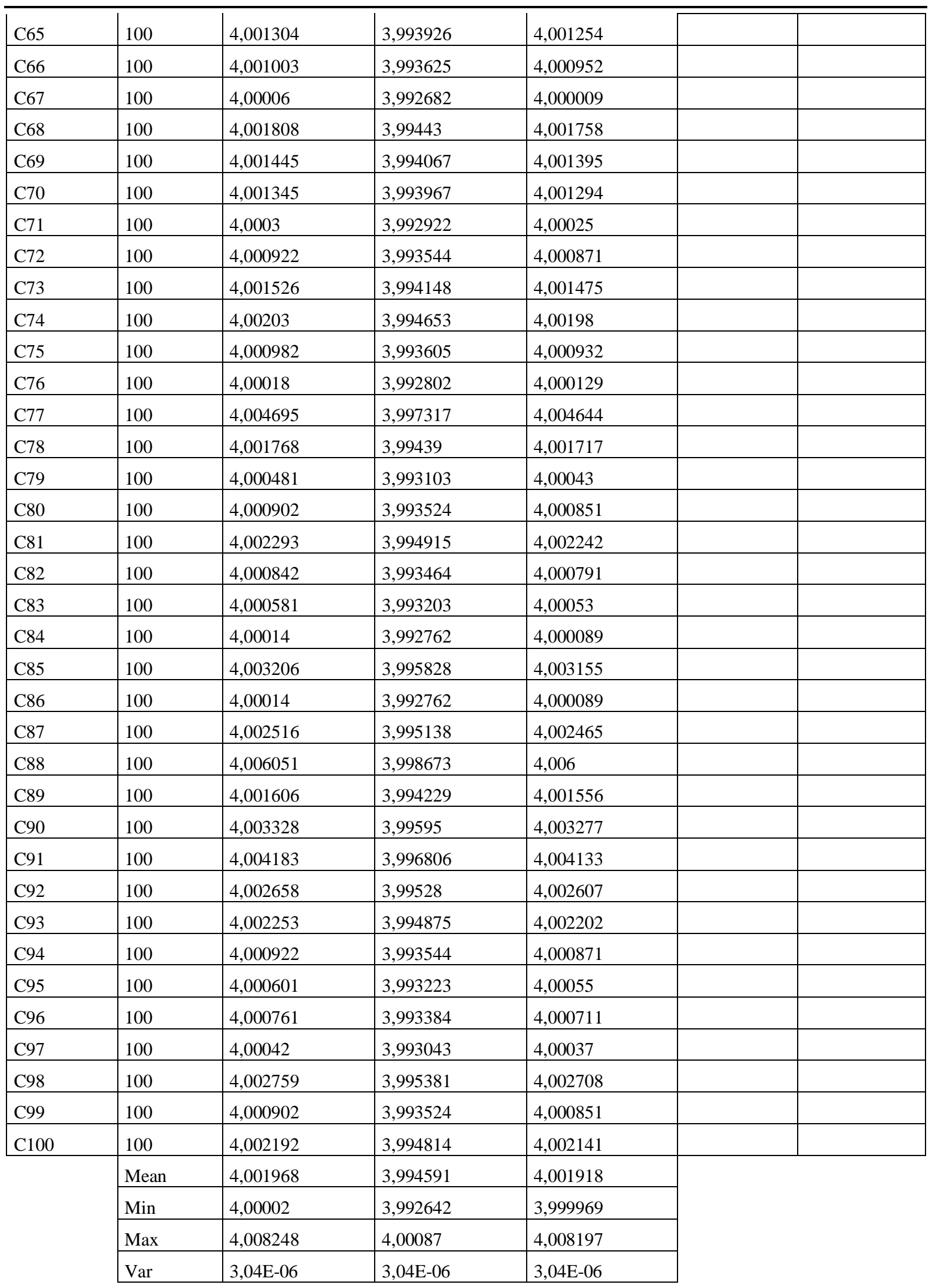

The null hypothesis $H_{0}: \beta=4$ is rejected in 3 out of 100 tests.

Three of the $95 \%$ confidence intervals exclude the threshold $\beta=4$. 